\title{
Increasing the capacity of natural killer (NK) cells in fighting advance stage ovarian cancer: A cellular immunotherapy minireview
}

\author{
Radiana Dhewayani Antarianto ${ }^{a b}$, Fransisca Dela Verna ${ }^{b}$, Lady Feren Pangjayabc, Sanya Khaerunnisabc, \\ Dinda Shezaria ${ }^{\mathrm{bc}}$, Tricia Dewi Anggraeni ${ }^{\mathrm{d}}$
}

aDepartment of Histology Faculty of Medicine, Universitas Indonesia

bStem cell and Tissue Engineering Research Cluster, IMERI, Universitas Indonesia

'Biology Undergraduate Program Faculty of Mathematics and Natural Science, Universitas Indonesia

dDivision of Oncology Gynecology Cipto Mangunkusumo National General Hospital, Department of Obstetric Gynecology, Faculty of

Medicine, Universitas Indonesia

\begin{abstract}
Global Cancer Statistics in 2018 estimated 300,000 new cases of ovarian cancer with 152,000 mortality rate each year. The Indonesian Society of Gynecologic Oncology reported 30\% of gynecologic cancer is ovarian cancer, which has a 125,000 mortality rate each year. Ovarian cancer data in Indonesia showed that $70 \%$ of patients were diagnosed with ascites or metastasis beyond the ovaries (stage III or IV). Ovarian cancer is an immunogenic disease with an immunotherapy intervention on the horizon. To assess the potency of stimulated NK cells as ovarian cancer cellular immunotherapy, literature search was collected from NCBI, ScienceDirect and Pubchem database. A total of 15 articles relevant to our search terms were included in this minireview. NK cells from ovarian cancer ascites exhibit low cytotoxic efficacy but can be restimulated using IL-2 or IL-15. An in vitro study that incubated NK cells with an IL-15 fusion protein enhanced the function of the ovarian cancer ascites' NK cells or the healthy NK cells against the ovarian cancer ascites cells. Human IL-12-, IL-15- and IL-18-induced memory like (CIML) NK cells, has been proven to increase the elimination of xenograft human ovarian cancer cells over a long period of time in a mouse model. CIML NK cells also showed higher NK cell expansion and an enhanced function in the ovarian cancer ascites' microenvironment, which was immunosuppressive. Phase I-II clinical trials on NK cell-based adoptive cellular therapies demonstrated limited clinical benefit. The major challenges are obtaining persistent NK cells with anticancer activity.
\end{abstract}

\author{
Article history: \\ Received 06 Jul 2020 \\ Revised 24 Aug 2020 \\ Accepted 25 Aug 2020 \\ Available online 31 Aug 2020 \\ Keywords: \\ NK cell \\ Ovarian cancer \\ Cellular immunotherapy \\ Indonesian Society of \\ Gynecologic Oncology

\begin{abstract}
*Corresponding author: Phone: +62-21-3146129, Fax: +62-21-3160108
\end{abstract} \\ radiana.dhewayani@ui.ac.id,
}

\section{Introduction}

Cancer poses a death threat with an unprecedented rise in developing countries. Globally, around $16 \%$ or 1 in 6 deaths are due to cancer, with $70 \%$ of cases occurring in developing countries (Reid et al., 2017). In Indonesia, cancer is the fifth-leading cause of death, with the number of cancer patients increasing from year to year. The Indonesian Ministry of Health states that cancer attacks 100 out of every 100,000 people, and the average number of women with cancer are about 2.2 per 1000 women (Aziz, 2009). A study conducted by the Global Cancer Observatory in 2012 estimated that there were 239,000 cases of and 152,000 deaths from ovarian cancer. This number is predicted to increase by $55 \%$ to 317,000 for ovarian cancer cases and deaths will increase by $67 \%$ to 254.000 by 2035 (Reid et al., 2017).

Several studies reported that the risk was increased in older women at menopause. The average age of women with an ovarian cancer diagnosis ranges from 45-54, and the five-year relative survival rate is around 54.8\% (Aziz, 2009). An increase in the death rate of ovarian cancer each year is caused by the slow administration of the diagnosis. About $70 \%$ of ovarian cancer patients are diagnosed at a late stage (Aziz, 2009).

Ovarian cancer is an immunogenic disease with immunotherapy intervention on the horizon. Although they play a key role in innate immune reactions and have the ability to kill tumour cells without requiring the introduction of a specific antigen, recent study results discovered that NK cells have the intrinsic capacity to develop adaptive immune system capabilities. This review addresses the use of NK cell stimulation as a potential cellular immunotherapy for fighting advanced stage ovarian cancer.

\section{Methods}

This systematic literature search was performed between April and June 2019 in three databases: NCBI, ScienceDirect and Pubchem. The combinations of terms used for the search included 'NK cell', 'ovary cancer', 'cellular immunotherapy' and 'Indonesian ovary cancer epidemiology'. The inclusion criteria were (i) studies published in the last 5 years, (ii) review article or original article and (iii) full text article with open access. Exclusion criteria was 
any criteria that did not meet the above standards. A total of 15 articles that met the criteria were acquired.

\section{NK cells and their involvement in cancer}

NK cells are regulated by activating and inhibitory signals that work on NK cell receptors. Inhibitory receptors play a role in preventing NK cells from attacking autologous normal cells while activating receptors help identify ligands expressed on the surface of a virus-infected cell or undergoing a transformation and enable the NK cells to kill the abnormal cells. The activation of NK cells is dependent on binding of ligands with NK cell activating receptor (NKR) including NKG2D; Natural cytotoxicity receptors (NCRs), such as NKp46 and NKp30; and NKp44 receptors. The inhibitory receptors on the NK cell include the HLA-I-specific inhibitory receptor, killer Ig-like receptors (KIRs) and CD94/NKG2A receptors. Research carried out by Han et al. in 2018 reported a correlation between the low percentage of NK-cell-activating-receptors NKp30, NKp46, NKG2D and DNAM - 1 , with an increase in TGF- $\beta 1$ in gastric carcinoma patients. Inhibition of the TGF- $\beta 1$ receptor showed the opposite result and revealed a potential target for gastric carcinoma therapy (Han et al., 2018).

The dynamic expression of NK cell receptors has been observed. Activation of NK cells begin with priming and ends with cytotoxic response. NKG2D and CD96 expressions have a positive correlation with each other, with the down-regulation of one activating receptor's expression being related to the downregulation of the other. The CD96 expression retention on the surface of the NK cell is important for the activation of the NK cell, which is measured by the CD96 expression. The NKG2D expression retention and the CD96 up-regulation on the cell surface of primed NK cells seems crucial to the TNF- $\alpha$ receptor's up-regulation. This finding indicates that NKG2D and CD96 are essential for NK cell priming, whereas activating receptor NKp46 is important for triggering the NK cell's cytotoxic response because it was found to have a correlation with the lysis of K562 and PC3 prostate cancer cells but no connection with the activation of NK cells (Hood et al., 2019).

Based on several studies, there are at least three determining factors of NK cell function: (1) the phenotypes and composition of the NK cell population at rest, (2) the stimulus type used to activate the NK cells (i.e. cytokines or membrane-bound target ligands) and (3) the stimulus effect on the current NK cell phenotype, which then determines the NK cell's ability to lyse targets. Both IL-2 stimulation and CTV-1 priming of NK cells creates an increasing proportion of NK cells expressing NKG2D, CD69, CD96, CD107a (degranulation receptor), CD137, OX40 and GITR (TNF receptors); however, CD16 expression, which was down-regulated with CTV-1 priming, was not affected by IL-2 stimulation. The activating receptor downregulation that occurs with priming was suspected to be the result of the exposure to the immunosuppressive cytokines secreted by the CTV-1 cell rather than the result of ligation with expressed ligands on the surface of the CTV-1 cells. The NK cell response to priming with K562 cells and CTV-1 cells showed a down-regulation of CD16 and up-regulation of CD69 (early activation marker), which is positively correlated with TNF- $\alpha$ receptor up-regulation (Hood et al., 2019).

Phenotype analysis based on NK receptor expressions consistently showed different subsets of NK cells or the heterogeneity of NK cell effector in the peripheral blood. NK cell development also governed self-tolerance and generated long-term memory NK cells. Around $40 \%$ of NK cells are derived from the lymphoid progenitor that expresses recombination activating gene (RAG). The role of RAG in the functional diversity of NK cells is during NK cell development and selective NK cell subset fitness. NK cell development begins in the bone marrow and has a higher percentage of RAG + NK cells. Further development of circulating
NK cells showed decreased RAG + NK cells in the peripheral blood. RAG- NK cells were related to more mature and activated NK cell phenotypes. Selective NK cell subset fitness, regulated by RAG expression, refers to the survival and expansion of NK cell subsets after subsequent antigen challenge, e.g. viral infection. RAGgenerated DNA breaks are important for the persistence of longlived NK cells in combating pathogens (Karo et al., 2014).

\section{NK cells and their role in ovarian cancer}

In 2018, Hoogstad-van Evert and co-workers observed the notable shift in the CD56dim/bright NK cell ratio when the NK cell ratio from a healthy donor were compared to NK cell ratio in the peritoneal fluid samples of patients with benign gynecological disorders (BGDs) and ovarian cancer. The benign ascites fluid samples showed a $32.4 \%$ CD56dim population and a $67.5 \%$ CD56bright population, whereas the malignant samples showed a $54.7 \%$ CD56dim population and a $45.4 \%$ CD56bright population (Hoogstad-van Evert et al., 2018).

Ascites-derived NK cells of advance stage ovary cancer patients with stage III C or IV and histopathology of high-grade serous papillary ovarian carcinoma were found to manifest lower expressions of activating receptors in comparison to the activating receptor expression levels of the benign gynecological disorder control group. The expression of NKG2D was low to undetectable on ascites-derived NK cells in ovarian carcinoma samples. Notably, NKp30 expression in benign peritoneal fluid samples were detected with an average of $79 \%$, whereas it was barely detected in ovarian carcinoma samples. Additionally, NKp46 and DNAM-1 expressions were found to be lower in malignant samples, particularly in patients with a low chance of survival (Hoogstad-van Evert et al., 2018).

\section{NK cells as alternative for immunotherapy}

IL-15 primed CD56bright NK cells have shown increasing levels of cytotoxic effector protein expression and increased cytotoxicity to tumour target cells. NK cell priming with IL-15 also significantly improves the degranulation and cytokine production of NK CD56bright cells in response to the K562 acute myeloid leukaemia (AML) cell line and the HLA class I-expressing HL-60 AML cell line, evident because of a noticeable increase in CD107a (LAMP1), IFN$\gamma$ and TNF- $\alpha$ production (Wagner et al., 2017).

Prior stimulation with IL-15 has proven to enhance the ability of in vitro cultured healthy donor NK cells for mediating immunologic effector functions in reaction to target cells, such as A1847, MA-148 and SKOV3 ovarian cancer cell lines, as shown by the increasing expression of $\mathrm{CD} 107, \mathrm{IFN} \gamma$ and TNF $\alpha$. In contrast, NK cells from healthy donors, when co-incubated with the same cancer cell line without prior IL-15 stimulation, only showed minor degranulation, assessed by measuring the expression of surface CD107a, and inflammatory cytokine production, assessed by measuring the expression of intracellular IFN $\gamma$ and TNF $\alpha$. These NK cells from healthy donors that have undergone IL-15 prior stimulation show increased proliferation, whereas the NK cells which are not stimulated show decreased proliferation and increased cell death. The proportion of CD16 activating receptor necessary for antibody-dependent cell-mediated cytotoxicity from ovarian cancer patient ascites is lower in comparison to the proportion from healthy donor peripheral blood (Felices et al., 2017).

Immunotherapy is one of the alternatives for eradicating cancer cells, as opposed to surgery, chemotherapy and radiotherapy (these last two have side effects). It is hoped that immunotherapy will be able to stimulate heightened activity of patient's immune system towards cancer microenvironments, such as ascites. The NK cell is a suitable candidate for adoptive immunotherapy because of its ability to kill target or tumour cells without prior sensitization. 
Treatment that uses NK cells is still new and in developmental stages. Nham et al increased the cytotoxic capacity of NK cells using artificial-antigen presenting cells and tested the NK cells against ovarian cancer ascites cells with a positive result (Nham et al., 2018).

Peripheral blood is one of the widely used sources for NK cells. Peripheral blood NK cells are able to kill tumour cells immediately; however, impaired NK cells are found in ovarian cancer ascites. Restimulation of these impaired NK cells could lead to recovered NK cell function. Ascites of late stage ovarian cancer patients were found to impair the function of the human immune system. These impairments directly affect NK cell function. Furthermore, there are plenty of NK cells inside the ascites fluid. The impairment causes a low cytotoxic capacity of the ascites' NK cells and is correlated with the phenotype of the ovarian cancer ascites' NK cells. The activating, inhibitory and maturity surface markers of the ascites' NK cells are different from the ovarian cancer peripheral blood and normal NK cells. In the Nham et al. study, an artificial antigen presenting cell (APC) were used to alter the phenotype and function of the NK cells of the ovarian cancer ascites. This study also developed NK cells ex vivo using an expansion technique that originates from ovarian cancer ascites. The artificial APC induced proliferation and activated the ovarian cancer ascites' NK cells. CD56dimCD16+ NK cells escalated to a CD56brightCD16+ phenotype after the ex vivo expansion. Subsequently, the expanded ovarian cancer ascites' NK cells, with CD56brightCD16+, effectively induced a tumour cell lysis and cytotoxic function in the ex vivo environment (Nham et al., 2018). Thus, the interaction between the engineered APC and the ovarian cancer ascites' NK cells upregulate the lytic capacity of NK cells.

In vivo NK cell re-stimulation was done using the incubation of NK cells with PM21 particle for PD-L1 induction. The study was performed to enhance the success rate of PD-1/PD-L1 blockade therapy. This targeted therapy is only relevant for patients with a PD-L1 positive tumour. PM21-NK cells can be transferred to induced PD-L1 expression in the tumour. Combination PM21-NK cells with anti-PD-1 blockade therapy could then be used to target the non PDL1 expressing tumour in addition to enhanced NK cell cytotoxicity (Oyer et al., 2018).

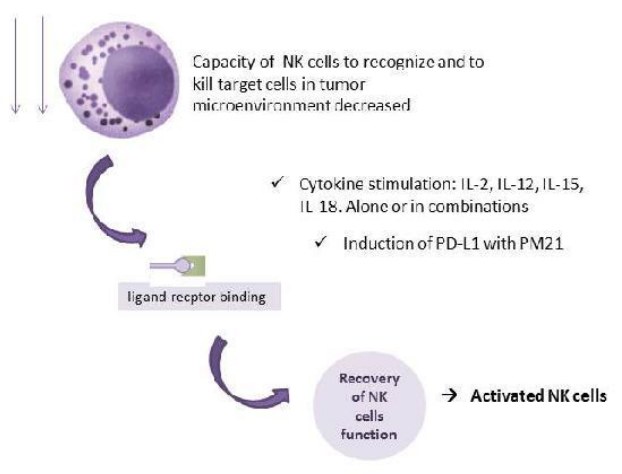

I MUNOTHERAPY $\rightarrow$ NKCELLS

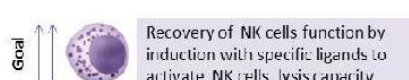
inductiun wilh specific lieands
activate NK Letlls lysiscapacity

Fig.1. Recapitulation of NK cells induction to achieve NK cell adoptive immunotherapy

Ovarian cancer cells were isolated from the patients, cultured, and then injected subcutaneously to a BALB/c mouse, which was conditioned as an immune deficient animal model. The human NK cells were isolated from peripheral blood mononuclear cells and cultured to be expanded in vitro. It was found that the NK cells have the capacity to inhibit ovarian cancer metastasis in vitro as well as in the mouse model. The transferred human NK cells showed the capacity to kill the ovarian cancer cell line and the induction of mouse CD4+ and CD8+ differentiation and reduce systemic metastasis. These data suggested NK cell potency as adoptive ovarian cancer immunotherapy (Sun et al., 2018). Another alteration of NK cell incubated with IL-12, IL-15 and IL-18 studied in a mouse model showed an increase in the elimination of xenograft human ovarian cancer cells. These interleukins will improve the IFN- $\gamma$ secretion of NK cells after being stimulated for weeks. The NK cells grow in culture and pass on these traits to the next NK cells sub-culture which will then produce cytokineinduced-memory (CIML) NK cells in 16 hours. These cells will be co-cultured with target cancer cells to observe the NK cells' function and phenotype. After the in vitro evaluation in an immunosuppressive microenvironment, the CIML NK cells were injected intraperitoneally into a xenogeneic ovarian cancer cell's mouse model. The results show that CIML NK cells elevate the IFN$\gamma$ production. CIML NK cells have more effector function compared to the normal NK cells in the immunosuppressive microenvironment. CIML NK cells also show potent antitumor effects to ovarian cancer cells in a mouse model. It can be noted that CIML NK cells have increased functionality and endurance against ovarian cancer cells (Uppendahl et al., 2018, 2019).

\section{The challenge of adoptive NK cell therapy}

Clinical trial phase I reported a well-tolerated response of allogeneic NK cells administered intravenously for patients with advanced or recurrent ovarian cancer. Another case report recorded a steep decline in ovarian cancer marker after six rounds of allogeneic NK cell intravenous infusion as a first-line therapy for an ovarian cancer patient (Aziz, 2009). Despite this early positive hint on adoptive NK cell immunotherapy, the ex vivo expansion of NK cells required before treatment and the priming and maintenance for the survival of transferred NK cells in vivo are current, active areas of research.

To regulate its expansion and function, NK cells require cytokines, including IL-2, IL-12 and IL-15. The level of these cytokines is considered as an indicator of NK cell adoptive therapy efficacy. Some evidence on NK cell adoptive therapy suggests that it seems to be a promising adjuvant therapy for ovarian cancer patients; however, several clinical trials showed a limited effectiveness of NK cell adoptive therapy. One of the phase II clinical trials showed that although IL-15 and IL-2 levels in patients serum increased after a lymphodepletion regimen compared to the baseline, it began to fall after two weeks of NK cell administration. Other clinical trials present evidence of toxicity, especially related to use of IL-2 to promote the expansion of NK cells, and a more severe degree of toxicities in patients with multi-resistant epithelial ovarian cancer, as evidenced by mortality due to tumour lysis syndrome, lymphocyte syndrome and autoimmune haemolytic anemia (Mittica et al., 2016). The attempt to harness NK cell antitumor activity for adoptive immunotherapy is an innovative alternative approach. Cytokine and other specific stimulations of NK cells may mediate higher NK cell cytotoxic functionality and persistence against ovarian cancer (Greppi et al., 2019).

Altogether, the studies reviewed above showed that enhancing NK cell cytotoxic responsiveness against tumour target cells can improve the efficiency of adaptive immunotherapy mediated NK cells and clinical outcomes of patients with malignancy. This is provided that the effector function of NK cells can be released once the requirements for NK cell activation are fulfilled.

A major challenge in adoptive cellular immunotherapy for ovarian cancer is obtaining persistent NK cells with anticancer activity. Thus, further studies are necessary to better understand the mechanism of NK cells' in vitro stimulation mechanism against ovarian cancer cells, in addition to discovering an effective protocol 
for increasing NK cell expansion, persistence and cytotoxic function for the purpose of clinically effective NK cell adoptive immunotherapy

\section{Conclusion}

Clinical trial phase showed limited benefit due to major challenges of obtaining NK cells with persistent anticancer activity. Further research is needed to induce NK cells for adoptive cell transfer.

\section{Acknowledgement}

Funding for this research was obtained from the Ministry of Research Technology and Higher Education Republic of Indonesia through the PDUPT Universitas Indonesia Research Grant 2019 contract number NKB-1560/UN2.R3.1/HKP.05.00/2019 with Radiana Dhewayani Antarianto as the principal investigator.

\section{Conflict of Interest}

The authors declare no conflict of interest regarding the material written and discussed in the manuscript.

\section{References}

Aziz MF. 2009. Gynecological cancer in Indonesia. J Gynecol Oncol 20(1): 8-10. doi: 10.3802/jgo.2009.20.1.8

Felices M, Kodal CB, Bendzick L, Ryan C, Lenvi AJ, Boylan KLM, Wong HC, Skubitz PN, Miller JS, Geller MA. 2017. IL-15 super-agonist (ALT-803) enhances natural killer (NK) cell function against ovarian cancer. Gynecol Oncol 145(3): 453-61. doi: 10.1016/j.ygyno.2017.02.028

Greppi M, Tabellini G, Patrizi O, Candiani S, Decensi A, Parolini S, Sivori S, Pesce S, Palerari L, Marcernaro E. 2019. Strengthening the antitumor NK cell function for the treatment of ovarian cancer. Int J Mol Sci 20(4): 89. doi: $10.3390 /$ ijms 20040890

Han B, Mao F-Y, Zhao Y-L, Lv Y-P, Teng Y-S, Duan M. et. al. 2018. Altered NKp30, NKp46, NKG2D, and DNAM-1 expression on circulating NK cells is associated with tumor progression in human gastric cancer. $J$ Immunol Res 2018: doi: 10.1155/2018/6248590

Hood SP, Foulds GA, Imrie H, Reeder S, Mcardle SEB, Khan M. 2019. Phenotype and function of activated natural killer cells from patients with prostate cancer: Patient-dependent responses to priming and IL-2 activation. Front Immunol 25(9): 3169. doi: 10.3389/fimmu. 2018.03169

Hoogstad-van Evert JS, Maas RJ, Meer J, Cany J, Steen S, Jansen JH, Miller JS, Bekkers R, Hobo W, Massuger L, Dolstra H. 2018. Peritoneal NK cells are responsive to IL-15 and percentages are correlated with outcome in advanced ovarian cancer patients. Oncotarget 9(78):34810-20. doi: 10.18632/oncotarget.26199

Karo JM, Schatz DG, Sun JC. 2014. The RAG Recombinase dictates functional heterogeneity and cellular fitness in natural killer cells. Cell 159(1): 94-107. doi: 10.1016/j.cell.2014.08.026

Mittica G, Capellero S, Genta S, Cagnazzo C, Agglieta M, Sangiolo D, Valabrega G. 2016. Adoptive immunotherapy against ovarian cancer. $J$ Ovarian Res 9: 30. doi:10.1186/s13048-016-0236-9

Nham T, Poznanski SM, Fan IY, Shenouda MM, Chew MV, Lee AJ, Vahedi F, Karimi Y, Butcher M, Lee DA, Hirte H, Ashkar AA. 2018. Ex vivoexpanded NK cells from blood and ascites of ovarian cancer patients are cytotoxic against autologous primary ovarian cancer cells. Cancer Immunol Immunother 67(4): 575-87. doi: 10.1007/s00262-017-2112-x

Oyer JL, Gitto SB, Altomare DA, Copik AJ. 2018. PD-L1 blockade enhances anti-tumor efficacy of NK cells. Oncoimmunology 7(11): e1509819. doi: $10.1080 / 2162402 X .2018 .1509819$

Reid BM, Permuth JB, Sellers TA. 2017. Epidemiology of ovarian cancer: a review. Cancer Biol Med 14(1): 9-32. doi: 10.2147/IJWH.S197604

Sun Y, Yao Z, Zhao Z, Xiao H, Mengting X, Xiaojun Z, Jiang X, Sun C. 2018. Natural killer cells inhibit metastasis of ovarian carcinoma cells and show therapeutic effects in a murine model of ovarian cancer. Exp Ther Med 16:1071-8. doi: 10.3892/etm.2018.6342

Uppendahl LD, Dahl CM, Miller JS, Felices M, Geller MA. 2018. Natural killer cell-based immunotherapy in gynecologic malignancy: A review. Front Immunol 8: 1825. doi: 10.3389/fimmu.2017.01825

Uppendahl LD, Felices M, Bendzick L, Ryan C, Kodal B, Hinderlie P, Boylan KLM, Skubitz APN, Miller JS, Geller MA. 2019. Cytokine-induced memory-like natural killer cells have enhanced function, proliferation, and in vivo expansion against ovarian cancer cells. Gynecol Oncol 153(1): 149-57. doi: 10.1016/j.ygyno.2019.01.006

Wagner JA, Rosario M, Romee R, Berrien-Elliott MM, Schneider SE, Leong JW, Sullivan RP, Jewell BA, Becker-Hapak M, Schapper T, Abdel-Latif S, Ireland AR, Jaishankar D, King JA, Vij R, Clement D, Goodridge J, Malmberg KJ, WOng HC, Fehniger TA. 2017. CD56bright NK cells exhibit potent antitumor responses following IL-15 priming. $J$ Clin Invest 127(11): 4042-58. doi: 10.1172/JCI90387 